\title{
RESOLUÇÃO DE DISFUNÇÃO PRECOCE GRAVE DE ENXERTO HEPÁTICO EM PACIENTE PEDIÁTRICO COM USO DE N-ACETILCISTEÍNA: RELATO DE CASO
}

\author{
$\mathrm{N}$-acetyl cysteine use in a Pediatric Patient with Severe Early Allograft Dysfuntion: \\ Case Report
}

\author{
Helry Luiz Lopes Cândido', Gabriel Guerra Cordeiro², Pedro Lukas do Rêgo Aquino, \\ Guilherme Lopes Cândido Honório3, Lígia Patrícia de Carvalho Éboli1, Paulo Sérgio Vieira de Melo', \\ Olival Cirilo da Fonseca Neto', Anderson André Pantoja Dias', Américo Gusmão Amorim¹, \\ Priscylla Jennie Monteiro Rabêlo', Norma Thomé Jucá1, Lara Neves Souza1, Cláudio Moura Lacerda
}

\section{RESUMO}

A disfunção precoce de enxerto hepático consiste em uma síndrome multifatorial caracterizada por alterações clínicas e laboratoriais associadas à alta morbidade nos primeiros dias pós-transplante. Relacionada à lesão de isquemia e reperfusão do fígado, pode levar em sua forma mais grave a alterações sistêmicas abruptas. Apesar de ser um quadro grave, as alterações podem ser transitórias, possibilitando recuperação do enxerto hepático. Sua ocorrência em população pediátrica é rara. A N-acetilcisteína, comumente utilizada no tratamento de hepatotoxicidade secundária à overdose por paracetamol, tem surgido como alternativa eficaz na melhora clínica de pacientes transplantados com má função de enxerto. Relatamos um caso de paciente do sexo masculino, dois anos, submetido a transplante de fígado por atresia de vias biliares, sem intercorrências intraoperatórias. Evoluiu com quadro de disfunção precoce grave de enxerto no segundo dia pós-operatório, sem boa resposta às medidas de suporte, cogitando-se indicação de retransplante. Foi iniciada administração de n-acetilcisteína, dosagem de 100mg três vezes ao dia, progredindo com melhora clínica significativa, sobretudo da função hepática. O acompanhamento de dados clínicos e laboratoriais de pacientes no período pós-transplante é fundamental, tendo em vista riscos e graves repercussões da disfunção precoce do enxerto hepático. A N-acetilcisteína é uma droga que tem sido relatada atuando no aumento da oferta de oxigênio tecidual e na remodelação dos hepatócitos, a partir da recuperação dos níveis de glutationa. Os efeitos em pacientes com disfunções hepáticas pós-transplante têm sido promissores nos poucos relatos científicos de seu uso, como o caso em questão.

Descritores: Acetilcisteína, Disfunção Primária do Enxerto, Sobrevivência de Enxerto, Transplante Hepático, Pediatria.

\section{Instituição:}

1 Unidade de Transplante de Fígado, Hospital Universitário Oswaldo Cruz - Universidade de Pernambuco - Pernambuco/PE

${ }^{2}$ Centro de Ciências Médicas, Hospital das Clínicas Universidade Federal de Pernambuco - Pernambuco/PE

${ }^{3}$ Curso de Medicina, Hospital Universitário Oswaldo Cruz Universidade de Pernambuco - Pernambuco/PE

\section{Correspondência:}

Gabriel Guerra Cordeiro

Email: gguerra.2607@gmail.com

https://doi.org/10.53855/bjt.v24i2.016

Recebido em 11/12/2020
Aceito em: 25/02/2021

\section{INTRODUÇÃO}

A atresia de vias biliares (AVB) é uma colangiopatia neonatal idiopática caracterizada por obstrução inflamatória progressiva dos ductos biliares intra ou extra-hepáticos. ${ }^{1}$ Fatores relacionados à evolução da doença podem justificar a maior frequência de hipoplasia de veia porta (VP) em portadores de AVB, tais como: esclerose e fibrose rapidamente progressivas, colangite de repetição e a hipertensão portal. ${ }^{1,2}$ A cirurgia de Kasai descrita em 1959, comporta-se como uma ponte para o transplante hepático, que é o tratamento definitivo. 
A técnica de Kasai consiste em uma portoenterostomia hepática, criando uma derivação biliodigestiva entre o porta hepatis e uma alça jejunal em Y-de-Roux. Entretanto, apenas 20 a $30 \%$ das crianças operadas pela técnica de Kasai tornam-se anictéricas após essa cirurgia e cerca de $90 \%$ das crianças ainda necessitarão de transplante de fígado. ${ }^{1-3}$

O transplante hepático deve ser indicado para casos selecionados, visto que, por mais que tenham havido avanços na técnica cirúrgica e inovações nas drogas imunossupressoras, muitas complicações graves ainda acontecem. Uma dessas complicações é a disfunção primária de enxerto (DPE), caracterizada por má função do enxerto hepático pós-transplante, sendo associada à alta mortalidade. Quanto mais jovem o receptor, mais rara é a ocorrência de DPE. ${ }^{3,4}$ A disfunção precoce do enxerto ( $E A D$ - early allograft dysfunction) e o não funcionamento primário (PNF - primary nonfunction) são divisões da DPE. ${ }^{2,3}$ A DPE ocorre de 5 a $36 \%$ dos transplantes hepáticos ${ }^{3}$ e sua evolução para PNF pode atingir até $8 \%$ dos casos. ${ }^{3-5}$

A N-acetilcisteína (NAC) é uma droga cujos mecanismos reais de atuação não são totalmente elucidados na área científica, mas seus efeitos nos pacientes com problemas hepáticos graves têm sido promissores em seus poucos relatos científicos de uso, como o caso em questão. ${ }^{6}$

O presente artigo objetiva descrever um caso de PNF em paciente pediátrico com melhora substancial através da administração de NAC, demonstrando a importância, pouco relatada na literatura, da aplicação desse medicamento como alternativa terapêutica para o referido quadro.

\section{RELATO DO CASO}

Paciente do sexo masculino, dois anos de idade, nascido de parto natural a termo, sem intercorrências, apresentando intensa icterícia neonatal no primeiro dia de vida, associada à colúria e acolia fecal. Não houve remissão do quadro mesmo após fototerapia. Foi realizada USG de abdômen, constatando-se hepatomegalia com textura heterogênea, ausência de dilatações biliares intra-hepáticas, espessamento ecogênico em região periportal e achados compatíveis com quadro de atresia de vias biliares. Com o diagnóstico, foi realizada portoenterostomia de Kasai no segundo mês de vida, porém, sem melhora do quadro colestático. Paciente foi alocado à lista de transplante hepático (TH) com escore PELD (Pediatric End-Stage Liver Disease) 39. Exames laboratoriais no pré-operatório evidenciaram: índice internacional normalizado (INR) - 1,18; aspartato aminotransferase (AST) - 223,57 U/L; alanina aminotransferase (ALT) - $203 \mathrm{U} / \mathrm{L}$; bilirrubinas total e direta (BT e BD), respectivamente - 17,46 e 16,19 $\mathrm{mg} / \mathrm{dl}$; desidrogenase láctica (DHL) - 284,6 U/L. Foi submetido a TH com um ano e 10 meses de vida, doador falecido, técnica cirúrgica convencional sem bypass e anastomose bílio-digestiva. Não houve síndrome pós-reperfusão do enxerto, com produção de bile no intraoperatório. Tempo de isquemia total do enxerto foi próximo de nove horas, sendo a isquemia fria de oito horas e dez minutos e a isquemia quente de 45 minutos.

Paciente evoluiu com disfunção precoce grave do enxerto, evidenciado no segundo dia pós-operatório (DPO), com exames registrando: INR - 8,15; AST - $7338 \mathrm{U} / \mathrm{L} ; A L T$ - $2799 \mathrm{U} / \mathrm{L} ; \mathrm{BD}$ - 9,38 mg/dl; BT $10,6 \mathrm{mg} / \mathrm{dl}$; DHL - $4102 \mathrm{U} / \mathrm{L}$. Houve piora do quadro, sendo cogitada indicação de retransplante. Realizouse biópsia do enxerto, evidenciando hepatopatia inflamatória subaguda, com alterações regenerativas e áreas de necrose (Figuras 1A e 1B). Diante do quadro, foi iniciada administração de $\mathrm{N}$-acetilcisteína $(100 \mathrm{mg})$, com dose inicial intravenosa e manutenção oral (8/8h), no segundo DPO. O paciente progrediu com melhora significativa do quadro sobretudo na função hepática, evidenciado no sétimo dia pós-operatório: INR - 3,22; ALT - 296,4U/L; AST: 72U/L. (Tabela 1). No seguimento de um ano pós-transplante, o paciente apresentou boa evolução, permanecendo sem queixas ou sinais de disfunção hepática.

\section{DISCUSSÃO}

As complicações do transplante hepático podem ser desde vasculares até disfunção primária do enxerto implantado, a qual pode se manifestar através da EAD ou da PNF. Desse modo, os esforços para o diagnóstico precoce dessa disfunção são extremamente necessários. ${ }^{7,8}$ Os estudos utilizam-se de diferentes parâmetros e pontos de corte para o diagnóstico de EAD. As alterações laboratoriais relacionadas com o metabolismo e a função hepática nos primeiros dias após o transplante são a base da suspeita diagnóstica. Desse modo, elevação sérica das enzimas transaminases, quadro de coagulopatia e redução da produção biliar são os principais indícios da EAD. Um dos critérios mais utilizados é o de Ploeg et al, $^{7}$ com mensuração do nível de transaminases e do tempo de protrombina, respectivamente. Neste estudo, a EAD é caracterizada por AST $>2.000 U \mathrm{U} / \mathrm{L}$, TP acima de 16 segundos e nível de amônia maior que $50 \mu \mathrm{mol} / \mathrm{L}$ entre o segundo e sétimo dia de pós-operatório.? 
Helry Luiz Lopes Cândido, Gabriel Guerra Cordeiro, Pedro Lukas do Rêgo Aquino, Guilherme Lopes Cândido Honório, Lígia Patrícia de Carvalho Éboli, Paulo Sérgio Vieira de Melo, Olival Cirilo da Fonseca Neto, Anderson André Pantoja Dias, Américo Gusmão Amorim, Priscylla Jennie Monteiro Rabêlo, Norma Thomé Jucá, Lara Neves Souza, Cláudio Moura Lacerda

Figura 1 - Cortes histológicos do enxerto hepático no $4^{\circ}$ dia pós-operatório, evidenciando difusa esteatose predominantemente microvesicular do parênquima, alterações regenerativas e pequenas áreas de necrose compatíveis com lesão de preservação grau 3.

$1 \mathrm{~A}$

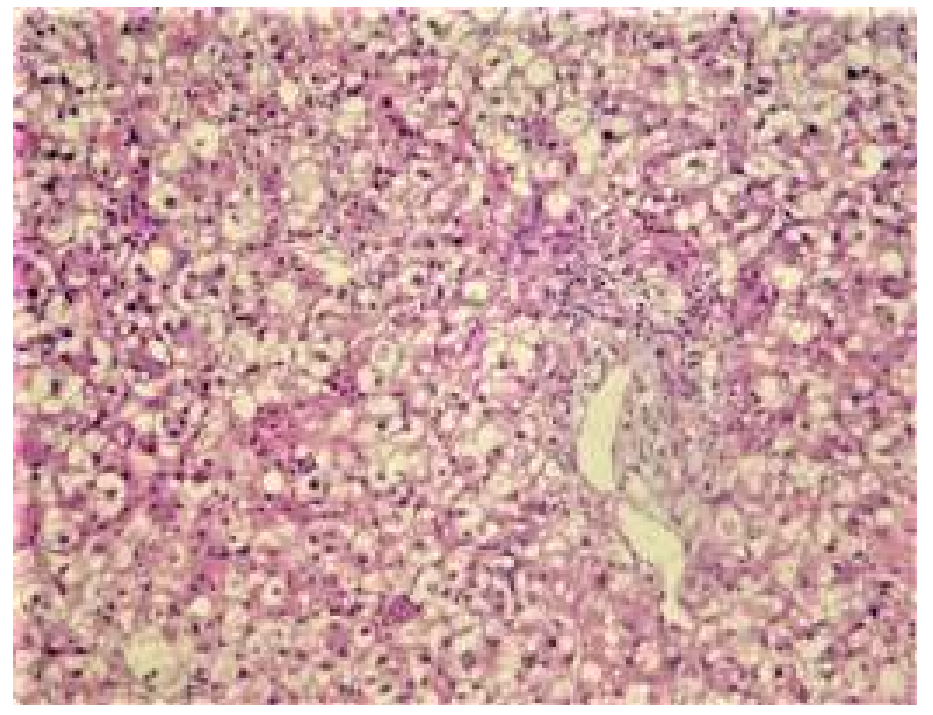

$1 \mathrm{~B}$

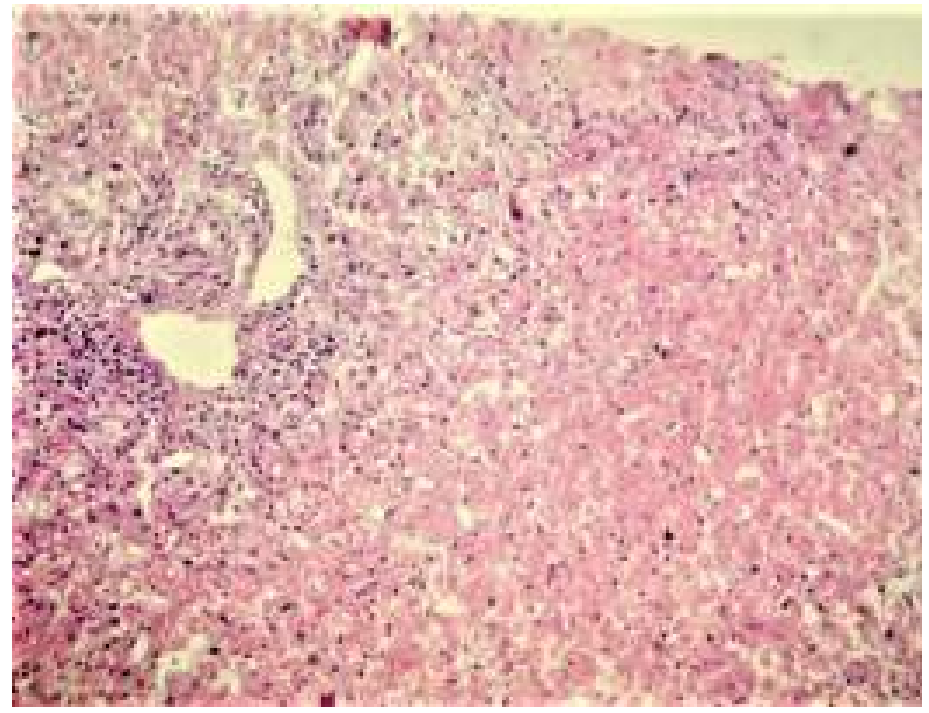

Tabela 1 - Evolução laboratorial pré e pós-transplante do paciente, indicando melhora importante do quadro com início da administração de $N$-acetilcisteína, sobretudo na função hepática

\begin{tabular}{|c|c|c|c|c|c|c|c|c|}
\hline & BT & BD & INR & AST & ALT & DHL & LAC & PQT \\
\hline Pré-operatório & 17,4 & 16,2 & 1,18 & 223,5 & 203 & 284 & 1,4 & 121 \\
\hline POI & 10 & 9,4 & 2,1 & 1944 & 777 & 2740 & 1,9 & 108 \\
\hline 10 DPO & 10,6 & 9,6 & 6,39 & 5688 & 2388 & 4102 & 2,1 & 78 \\
\hline 2 DPO & 11,1 & 9,4 & 8,15 & 7388 & 2799 & 3079 & 3,8 & 72 \\
\hline 3 DPO & 12,5 & 10,1 & 8,42 & 7787 & 2435 & 881 & 4,7 & 97 \\
\hline 4 DPO & 11,2 & 9,0 & 8,84 & 2110 & 931 & 514 & 5,5 & 105 \\
\hline 5 DPO & 9,67 & 7,96 & 3,5 & 361 & 566 & 320 & 3,4 & 98 \\
\hline 70 DPO & 7,93 & 6,85 & 3,22 & 72 & 296 & 283 & 1,7 & 118 \\
\hline $10 \circ \mathrm{DPO}$ & 5,36 & 4,44 & 1,39 & 33,1 & 59,3 & 185 & 0,9 & 153 \\
\hline 6 meses PO & 0,55 & 0,27 & 1,15 & 44 & 54 & 251 & - & 148 \\
\hline 1 ano $P O$ & 0,66 & 0,32 & 1,06 & 35,8 & 40,6 & 246 & - & 162 \\
\hline
\end{tabular}

Legenda: POI: Pós-operatório Imediato; DPO: Dia Pós-operatório; PO: Pós-operatório; BT: Bilirrubina Total; BD: Bilirrubina Direta; INR: Índice Internacional Normalizado; AST: Aspartato Aminotransferase; ALT: Alanina Aminotransferase; DHL: Desidrogenase Láctica; UR: Ureia; CRT: Creatinina; FBN: Fibrinogênio; PQT: Contagem de Plaquetas (10³). 
O PNF é uma condição evoluída e mais grave da EAD que resulta em retransplante precoce ou óbito. Algumas manifestações clínicas dessa fase mais avançada são: encefalopatia hepática, hipercalemia, acidose metabólica e insuficiência renal oligúrica. ${ }^{8} \mathrm{Um}$ dos critérios de diagnóstico da PNF difundido é o da United Network for Organ Sharing (UNOS) sendo eles AST $\geq 3.000$, associado a pelo menos um dos seguintes: INR $\geq 2.5$; acidose, caracterizada por $\mathrm{pH}$ arterial $\leq 7,30$ ou $\mathrm{pH}$ venoso $\leq 7,25$ e/ou lactato $\geq 4 \mathrm{mMol} / \mathrm{L}$. Assim, no caso descrito, o paciente estava fazendo um quadro compatível com PNF por apresentar valor de AST de 7338 u/l e INR de 8,15, segundo os critérios da UNOS.

A fisiopatologia da EAD encontra-se centrada, principalmente, na isquemia sofrida pelo enxerto na cirurgia de captação do órgão, após clamp da artéria aorta. Após esse período, surge o período de isquemia fria, na qual o enxerto será conservado sobre temperaturas baixas, entretanto, haverá falta de nutrição celular. ${ }^{9,10}$ Apesar da falta de nutrição celular, o órgão exposto à isquemia fria mantém inalterado seu aspecto macroscópico. Na fase de reperfusão hepática na cirurgia do receptor pode ocorrer síndrome de reperfusão, caracterizada por alterações bioquímicas, resultantes da hipóxia persistente das células endoteliais. Isso causa formação de radicais livres que danificam as células do endotélio hepático e ativam as células de Kupffer, resultando em alterações nos níveis de proteínas associadas a eventos inflamatórios e imunológicos. ${ }^{9}$ Os achados do exame histopatológico incluem infiltrado inflamatório agudo, dano hepatocelular, como necrose coagulativa e grandes infiltrados de neutrófilos, que caracterizam a disfunção do enxerto. ${ }^{11}$ No caso relatado, o histopatológico evidenciou hepatopatia inflamatória subaguda, reforçando-se a síndrome de disfunção primária do enxerto.

Algumas drogas parecem ajudar na recuperação da DPE e foram usadas em estudos, como por exemplo a prostaglandina E1 (PGE1), prostaglandina ${ }^{12}$ e óxido nítrico (NO). ${ }^{12-14}$ PGE1 promete evitar insuficiência hepática fulminante, revertendo insuficiência hepática aguda em recém-transplantados. ${ }^{12}$ Foley et al ${ }^{15}$ administraram PGE1 (0,2-0,8 mg / kg por hora), em associação com outros fármacos para os receptores durante transplante de fígado e descobriram que essas drogas impediam a evolução para PNF e melhoraram os resultados clínicos. Outros autores também mostraram benefícios em seu estudo com o uso dessa droga. ${ }^{12}$ Entretanto, uma meta-análise não produziu evidência de que a administração de pacientes com PGE1 na DPE reduz o risco de morte ou necessidade de re-transplante de fígado. ${ }^{16}$ Desse modo, o efeito de PGE1 no PNF merece maior investigação. Acredita-se que o PGI2 desempenhe um papel superior ao PGE1, com respeito à inibição da agregação plaquetária, e esse ácido graxo também inibe a produção de citocinas. ${ }^{13}$

O uso da $\mathrm{N}$-acetilcisteína (NAC) endovenosa é padrão para tratamento da insuficiência hepática aguda associada à intoxicação pelo acetaminofeno. ${ }^{17}$ Entretanto, as outras causas de insuficiência hepática aguda, como na disfunção precoce grave de enxerto no pós-operatório de transplantes hepáticos, o uso da NAC ainda não é difundido. Alguns estudos sugerem que a $\mathrm{N}$-acetilcisteína também possa beneficiar pacientes com outras formas de insuficiência hepática aguda, através do aumento do fluxo sanguíneo ao fígado, bem como aumento da oferta de oxigênio ao órgão. ${ }^{17-19}$ Foley et al mostraram benefícios com o uso da droga em associação com outros fármacos, na dosagem de $6 \mathrm{~g}$ em receptores de transplante hepático. ${ }^{15}$ Porém, ainda não há ensaios clínicos que comprovem a melhora do prognóstico nesses casos. No caso descrito, a NAS mostrou-se efetiva, segura e sem notórios efeitos adversos, ressaltando sua importância como alternativa terapêutica.

A NAC é um precursor acetilado do aminoácido $L \square$ cisteína, tendo sido relatada atuando no aumento da oferta de oxigênio tecidual e na remodelação dos hepatócitos, a partir da recuperação dos níveis de glutationa depletados na fase de isquemia fria. Essa redução dos estoques de glutationa dificulta a capacidade hepática de superar o estresse oxidativo da reperfusão. ${ }^{18}$ A capacidade da NAC agir de forma análoga à glutationa, reagindo com os radicais livres através dos grupos sulfidrila ou como substrato para a sua síntese tem originado diversos estudos que buscam elucidar a sua eficácia na prevenção e tratamento das lesões associadas à formação de radicais livres em vários órgãos, especialmente nos fenômenos envolvendo isquemia e reperfusão. Tais fenômenos encontram-se presentes na maioria dos procedimentos cirúrgicos de grande porte que envolvem, sobretudo, a isquemia celular, tais como manobra de Pringle: oclusão temporária do hilo hepático para controle de hemorragia no trauma de fígado, de forma total ou parcial nas ressecções hepáticas e nos casos de transplante de fígado. $^{18}$ Modarresi et al apresentou em seu estudo benefícios da administração precoce da NAC na lesão renal tubular aguda pós-transplante de rim, através da medição de um marcador de lesão renal aguda precoce, lipocalina urinária associada a gelatinase e neutrófilos (u-NGAL), tendo obtido melhora da função do enxerto. ${ }^{19}$ 
Helry Luiz Lopes Cândido, Gabriel Guerra Cordeiro, Pedro Lukas do Rêgo Aquino, Guilherme Lopes Cândido Honório, Lígia Patrícia de Carvalho Éboli, Paulo Sérgio Vieira de Melo, Olival Cirilo da Fonseca Neto, Anderson André Pantoja Dias, Américo Gusmão Amorim, Priscylla Jennie Monteiro Rabêlo, Norma Thomé Jucá, Lara Neves Souza, Cláudio Moura Lacerda

Em uma revisão integrativa da literatura, Salviano et al, em 2019, mostraram que a NAC é uma droga segura e sem efeitos adversos significativos nos receptores de transplante renal, entretanto não conseguiram demostrar sua eficácia sobre os biomarcadores urinários. ${ }^{20}$

Com base nas evidências consolidadas da NAC para uso na insuficiência hepática aguda por intoxicação pelo acetaminofeno, bem como seu metabolismo antioxidante descrito na literatura, ${ }^{18}$ foi usada no tratamento da disfunção precoce grave de enxerto hepático do caso descrito. A dose usada foi de $100 \mathrm{mg} /$ $\mathrm{kg}$, via oral, com manutenção de $8 / 8 \mathrm{~h}$, a mesma preconizada nos casos de insuficiência hepática aguda por acetaminofeno. ${ }^{6,17} \mathrm{~A}$ droga é feita mais comumente por via intravenosa, entretanto Woodhead et al, em 2018, também demostraram efetividade da droga por via oral no tratamento de overdose por acetaminofeno e, assim, optou-se pela via oral no caso relatado. ${ }^{14}$

\section{CONCLUSÃO}

A identificação precoce e o acompanhamento adequado dos dados clínicos e laboratoriais em quadros de DPE grave são determinantes para um bom prognóstico dos pacientes. A NAC, fármaco amplamente utilizado no tratamento de overdose por acetaminofeno, também tem se mostrado eficaz no tratamento da DPE grave. Esse medicamento permite a remodelação e proteção dos hepatócitos, a partir da recuperação dos níveis de glutationa no fígado, tornando o enxerto menos susceptível ao estresse oxidativo pós-reperfusão.

Tal droga contribuiu para a resolução do caso descrito, tendo se apresentado segura, satisfatória e sem desencadear notórios efeitos adversos. À vista disso, a administração da NAC surge como alternativa terapêutica, que parece ser promissora em casos de má função do fígado nos primeiros dias pós-transplante.

\section{ABSTRACT}

Early allograft dysfunction consists of a multifactorial syndrome characterized by clinical and laboratory abnormalities associated to high morbidity in the early postoperative period. As to liver ischemia and reperfusion injury, in its most severe form it can lead to sudden systemic changes. Despite being a serious condition, it may be transient, allowing recovery of the liver graft. Its occurrence in pediatric population is rare. $\mathrm{N}$-acetylcysteine, commonly used to treat hepatotoxicity secondary to paracetamol overdose, has emerged as an effective alternative in the clinical improvement of transplant results in patients with poor graft function. Patient was two-year-old male, who underwent liver transplantation due to biliary atresia with no intraoperative complications. The patient evolved with severe early graft dysfunction on the second postoperative day, with no good response to supportive procedures, and indication for retransplantation has been considered. Administration of $n$-acetylcysteine started with a $100 \mathrm{mg}$ dosage three times a day, progressing with significant clinical improvement, especially in the liver function of the patient. Clinical and laboratory monitoring in the post-transplant period is essential, due to the risks and serious repercussions of early liver graft dysfunction. $\mathrm{N}$-acetylcysteine is a drug reported to act increasing the supply of tissue oxygen and in remodeling hepatocytes from the recovery of the glutathione levels. Effects in patients with post-transplant liver dysfunction have been promising in few scientific reports as to its use, as the case in question.

Keywords: Acetylcysteine, Primary Graft Dysfunction, Graft Survival, Liver Transplant, Pediatrics. 


\section{REFERÊNCIAS}

1. Jaw TS, Kuo YT, Liu GC, Chen SH, Wang CK. MR cholangiography in the evaluation of neonatal cholestasis. Radiology. 1999;212:249-56.

2. Silveira TR. Icterícia colestática neonatal. In: Miura E, Procianoy RS, editores. Neonatologia: princípios e prática. $2^{a}$ ed. Porto Alegre: Artes Médicas; 1977.p.196-212.

3. Uemura T, Randall HB, Sanchez EQ, Ikegami T, Narasimhan G, McKenna GJ, et al. Liver retransplantation for primary nonfunction: analysis of a 20 -year single-center experience. Liver Transpl. 2007;13(2):227-33.

4. Chen $X B, X u M Q$. Primary graft dysfunction after liver transplantation. Hepatobiliary Pancreat Dis Int. 2014;13(2):125-37. Review.

5. Lee WM et al Intravenous N-Acetylcysteine Improves Transplant-Free Survival in Early Stage NonAcetaminophen Acute Liver Failure. Gastroenterology. 2009;137:856-64.

6. Harrison PM, Wendon JA, Gimson AE, et al. Improvement by acetylcysteine of hemodynamics and oxygen transport in fulminant hepatic failure. N Engl J Med. 1991;324:1852-7.

7. Ploeg RJ, D'Alessandro AM, Knechtle SJ, Stegall MD, Pirsch JD, Hoffmann RM, et al. Risk factors for primary dysfunction after liver transplantation--a multivariate analysis. Transplantation. 1993;55(4):807-13.

8. Chen $X B, X u M Q$. Primary graft dysfunction after liver transplantation. Hepatobiliary Pancreat Dis Int. 2014;13(2):125-37. Review

9. Cortes M, Pareja E, García-Cañaveras JC, Donato MT, Montero S, Mir J, et al. Metabolomics discloses donor liver biomarkers associated with early allograft dysfunction. J Hepatol. 2014;61(3):564-74.

10. Chu MJ, Dare AJ, Phillips AR, Bartlett AS. Donor hepatic steatosis and outcome after liver transplantation: a systematic review. J Gastrointest Surg. 2015;19(9):171324.

11. Ricca L, Lemoine A, Cauchy F, Hamelin J, Sebagh M, Esposti DD, et al. Ischemic postconditioning of the liver graft in adult liver transplantation. Transplantation. 2015;99(8):1633-43.
12- Takaya S, Doyle H, Todo S, Irish W, Fung JJ, Starzl TE. Reduction of primary nonfunction with prostaglandin E1 after clinical liver transplantation. Transplant Proc 1995;27:1862-7.

13. Bärthel E, Rauchfuss F, Hoyer H, Habrecht O, Jandt K, Götz M, et al. Impact of stable PGI2 analog iloprost on early graft viability after liver transplantation: a pilot study. Clin Transplant 2012;26:E38-47

14. Woodhead, K., \& Foex, B. A. (2018). BET 1: In paracetamol overdose, is oral $\mathrm{N}$-acetylcysteine as effective as intravenous $\mathrm{N}$-acetylcysteine? Emergency Medicine Journal, 35(10), 643.2-645. doi:10.1136/ emermed-2018-208093.2 .

15. Foley DP, Fernandez LA, Leverson G, Chin LT, Krieger N, Cooper JT, et al. Donation after cardiac death: the University of Wisconsin experience with liver transplantation. Ann Surg. 2005;242:724-31.

16. Cavalcanti AB, De Vasconcelos CP, Perroni de Oliveira M, Rother ET, Ferraz L Jr. Prostaglandins for adult liver transplanted patients. Cochrane Database Syst Rev 2011;9: CD006006

17. Harrison PM, Wendon JA, Gimson AE, et al. Improvement by acetylcysteine of hemodynamics and oxygen transport in fulminant hepatic failure. $\mathrm{N}$ Engl J Med. 1991;324:1852-7.

18. Walsh TS, Hopton P, Philips BJ, et al. The effect of $\mathrm{N}$-acetylcysteine $\mathrm{n}$ oxygen transport and uptake in patients with fulminant hepatic failure. Hepatology. 1998;27:1332-40.

19. Modarresi, A., Nafar, M., Sahraei, Z., Salamzadeh, J., Chaibakhsh, S., Ziaie, S., ... Einollahi, B. (2018). $\mathrm{N}$-acetylcysteine decreases urinary level of neutrophil gelatinase-associated lipocalin in deceased-donor renal transplant recipients: a randomized clinical trial. Biomarkers, 23(6), 589-596. doi:10.1080/135475 0x.2018.1468823

20. Salviano, M. E. M., Lima, A. S., Tonelli, I. S., Correa, H. P., \& Chianca, T. C. M. (2019). Disfunção e não função primária do enxerto hepático: revisão integrativa. Revista Do Colégio Brasileiro de Cirurgiões, 46(1). doi:10.1590/0100-6991e-20192039 
Helry Luiz Lopes Cândido, Gabriel Guerra Cordeiro, Pedro Lukas do Rêgo Aquino, Guilherme Lopes Cândido Honório, Lígia Patrícia de Carvalho Éboli, Paulo Sérgio Vieira de Melo, Olival Cirilo da Fonseca Neto, Anderson André Pantoja Dias, Américo Gusmão Amorim, Priscylla Jennie Monteiro Rabêlo, Norma Thomé Jucá, Lara Neves Souza, Cláudio Moura Lacerda

\section{AGRADECIMENTOS}

Agradecemos à equipe multidisciplinar da Unidade de Transplante de Fígado de Pernambuco, que se dedica continuamente e de maneira inspiradora ao cuidado dos pacientes, incluindo o do caso apresentado. 